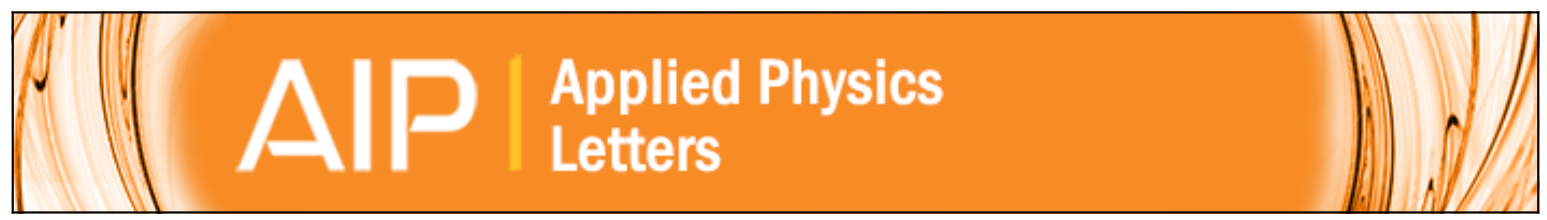

\title{
Tunable optical gratings based on buckled nanoscale thin films on transparent
} elastomeric substrates

Cunjiang Yu, Kevin O’Brien, Yong-Hang Zhang, Hongbin Yu, and Hanqing Jiang

Citation: Applied Physics Letters 96, 041111 (2010); doi: 10.1063/1.3298744

View online: $\mathrm{http}: / / \mathrm{dx}$. doi.org/10.1063/1.3298744

View Table of Contents: http://scitation.aip.org/content/aip/journal/apl/96/4?ver=pdfcov

Published by the AIP Publishing

\section{Articles you may be interested in}

Schottky-type surface plasmon detector with nano-slit grating using enhanced resonant optical transmission J. Appl. Phys. 116, 084313 (2014); 10.1063/1.4894150

Low-power and ultrafast all-optical tunable plasmon-induced transparency in plasmonic nanostructures Appl. Phys. Lett. 102, 201119 (2013); 10.1063/1.4807765

Resonant transmission of light through $\mathrm{ZnO}$ nanowaveguides in a silver film Appl. Phys. Lett. 101, 081113 (2012); 10.1063/1.4747718

Nanopattern enabled terahertz all-optical switching on vanadium dioxide thin film Appl. Phys. Lett. 98, 071105 (2011); 10.1063/1.3553504

Midinfrared filters based on extraordinary optical transmission through subwavelength structured gold films J. Appl. Phys. 106, 124313 (2009); 10.1063/1.3272716 


\title{
Tunable optical gratings based on buckled nanoscale thin films on transparent elastomeric substrates
}

\author{
Cunjiang Yu, ${ }^{1}$ Kevin O’Brien, ${ }^{2}$ Yong-Hang Zhang, ${ }^{2}$ Hongbin $\mathrm{Yu}^{2}$ and Hanqing Jiang ${ }^{1, a)}$ \\ ${ }^{1}$ School of Mechanical, Aerospace, Chemical and Materials Engineering, Arizona State University, \\ Tempe, Arizona 85287, USA \\ ${ }^{2}$ Center for Nanophotonics and School of Electrical, Computer and Energy Engineering, \\ Arizona State University, Tempe, Arizona 85287, USA
}

(Received 17 December 2009; accepted 4 January 2010; published online 29 January 2010)

\begin{abstract}
This letter reports a tunable optical grating based on buckled thin film with periodic sinusoidal patterns on a transparent elastomeric substrate. Submicron scale sinusoidal gratings have been fabricated with nanometer thick Gold/Palladium film coated on 30\% pretensioned polydimethylsiloxane substrates. Due to competition between the soft elastomeric substrates and relatively stiff films, periodic wavy profiles are created upon releasing the pretension. The buckling profiles can be easily tuned by mechanically stretching or compressing. Optical transmittance diffraction testing has been conducted, and $85 \mathrm{~nm}$ peak wavelength shifts of the first order diffraction have been achieved by stretching the grating up to $30 \%$ of its original length.

(C) 2010 American Institute of Physics. [doi:10.1063/1.3298744]
\end{abstract}

Diffraction gratings are key components in many applications such as optical telecommunications and spectroscopy. ${ }^{1,2}$ The application of the grating becomes extended and more interesting when the gratings can be externally tuned. A lot of efforts to date have been focused primarily on tunable gratings based on hard materials by multiple steps of micromachining technology. Some examples include electrostatically actuated suspended ribbons forming grating surfaces, ${ }^{3}$ complex comb drives driven diffraction gratings, ${ }^{4}$ and piezoelectric-driven tunable gratings. ${ }^{2}$ Another report regards using sinusoidal profile from compressing a polymer film as grating, however, only a change in the grating amplitude rather than the period was reported to tune the intensity. ${ }^{5}$ In this letter, we propose a simple method to fabricate the tunable gratings by harnessing the buckled nanoscale stiff thin films supported by elastomeric substrates. The buckled sinusoidal topography of the stiff thin film functions as the diffraction grating. The tunability of the diffraction grating is realized by reversibly changing the grating period or buckled wavelength by mechanical stretch and compression.

Ordered buckling structures of stiff thin films on elastomeric substrates, first reported by Bowden et al., ${ }^{6}$ have broad applications such as stretchable electronic interconnects ${ }^{7}$ and stretchable electronic devices, ${ }^{8-10}$ modern metrology methods, ${ }^{11}$ and methods for microfabrication/ nanofabrication. ${ }^{12}$ One way to achieve this buckling is to deposit $^{6}$ or transfer ${ }^{8}$ stiff thin films onto a prestrained elastomeric substrate followed by the relaxation of the prestrained substrate, which leads to buckled patterns in the stiff thin films with well defined wavelength and amplitude. The physical mechanism behind this is that the stiff thin films tend to buckle to release the compressive strain imposed by the relaxation of the prestretched substrate. Energetically, the bending energy due to the out-of-plane deformation (buckle) of the thin films reduces the total energy in the thin film/ substrate system. Mechanics models have been developed to

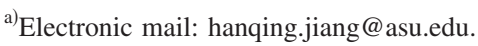

understand these systems using the energy method. ${ }^{13-15}$ The buckling period and amplitude are related to the prestrain by ${ }^{15}$

$$
\begin{aligned}
& d=\frac{2 \pi h_{\mathrm{f}}}{\left(1+\varepsilon_{\mathrm{pre}}\right)(1+\xi)^{1 / 3}}\left[\frac{E_{\mathrm{f}}\left(1-\nu_{\mathrm{s}}^{2}\right)}{3 E_{\mathrm{s}}\left(1-\nu_{\mathrm{f}}^{2}\right)}\right]^{1 / 3}, \\
& A=\frac{h_{\mathrm{f}}}{\sqrt{1+\varepsilon_{\mathrm{pre}}}(1+\xi)^{1 / 3}} \sqrt{\frac{\varepsilon_{\mathrm{pre}}}{\varepsilon_{\mathrm{c}}}-1,}
\end{aligned}
$$

where $\xi=5 / 32\left[\varepsilon_{\text {pre }}\left(1+\varepsilon_{\text {pre }}\right)\right]$ represents the large deformation and geometrical nonlinearity in the substrate and $\varepsilon_{\mathrm{c}}$ $=1 / 4\left[3 E_{\mathrm{s}}\left(1-\nu_{\mathrm{f}}^{2}\right) / E_{\mathrm{f}}\left(1-\nu_{\mathrm{s}}^{2}\right)\right]^{2 / 3}$ denotes the critical buckling strain or the minimum strain needed to achieve buckling. $E$ is the Young's modulus; $\nu$ is the Poisson's ratio, and the subscripts "s" and "f" refer to the substrate and stiff thin film, respectively. $h_{\mathrm{f}}$ is the thickness of the stiff thin film. If typical metallic or crystalline materials $\left(E_{\mathrm{f}}\right.$ is on the order of 100 $\mathrm{GPa})$ taken as the stiff thin films and polydimethylsiloxane (PDMS) $\left(E_{\mathrm{s}}\right.$ is about $\left.1 \mathrm{MPa}\right)$ as the elastomeric substrate, the buckling wavelength is about two orders higher than the thickness of thin film, with reasonable choice of prestrain.

The periodic buckling pattern provides a means for the diffraction properties. The simple law of diffraction can be expressed by the grating equation ${ }^{16}$

$$
n \sin \left(\theta_{m}\right)-n_{\mathrm{i}} \sin \left(\theta_{\mathrm{i}}\right)=\frac{m \lambda}{d},
$$

where $\theta_{\mathrm{i}}$ and $\theta_{m}$ are the angles of incidence and the $m^{\text {th }}$ diffraction order, respectively; $n_{\mathrm{i}}$ is the refraction index of the incident medium; $n$ is the refraction index of the medium where the diffracted orders propagate; $\lambda$ denotes the wavelength of the incident light; and $d$ is the period of the grating. For example, for $n=n_{\mathrm{i}}=1$ as air, $\theta_{\mathrm{i}}=0$, the first order diffraction spectra of visible light whose wavelength ranges from 380 to $760 \mathrm{~nm}$ would disperse at over an angular range from $22.3^{\circ}$ to $49.5^{\circ}$ for a grating period of $1 \mu \mathrm{m}$; while the spectra would occupy a smaller range of angles from $2.1^{\circ}$ to $4.4^{\circ}$ for a grating of $10 \mu \mathrm{m}$ period, which makes it difficult to 


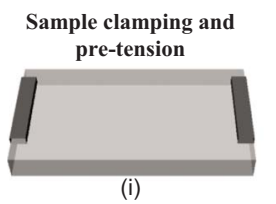

Oxygen plasma treatment and $\mathrm{Au} / \mathrm{Pd}$ sputter coating $N N N N N$

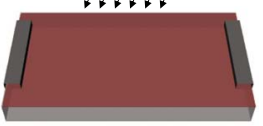

(ii)

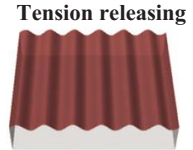

(iii)

(a)

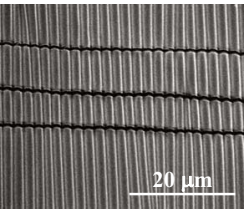

(b)

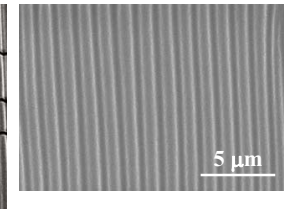

(c)

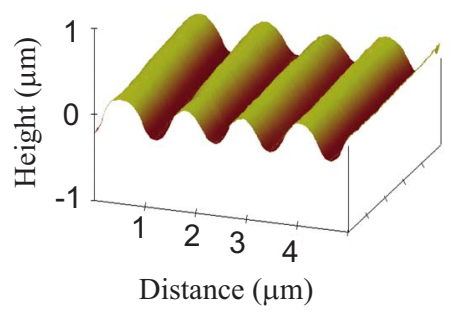

(c)
FIG. 1. (Color online) (a) Schematic illustration of the fabrication process. [(b) and (c)] SEM images of buckling profile of the Au/Pd thin films on PDMS substrates, (c) AFM image.

distinguish the spectrum. Therefore, to achieve substantial diffraction for the visible light range, submicron scale period of the wrinkling shape grating is desired.

According to the above analysis, to generate wrinkle patterns with submicron period $(d \sim 1 \mu \mathrm{m})$, the thickness of stiff thin film should be two orders smaller, i.e., $h_{\mathrm{f}}$ is on the order of $10 \mathrm{~nm}$, which is achieved by the following procedure shown in Fig. 1(a). The transparent PDMS substrates were prepared by casting the mixture of base and curing agent at the ratio of 10:1 by weight, cured, and cut to desired sizes. A $1 \mathrm{~mm}$ thick PDMS strip $\left(10 \times 40 \mathrm{~mm}^{2}\right)$ after oxygen plasma treatment (50 Watt, $1 \mathrm{~min}$ ) was prestretched by a custom made stage at desired prestrain. An ultrathin gold and palladium ( $\mathrm{Au} / \mathrm{Pd})(95 \% / 5 \%)$ film about $11 \mathrm{~nm}$ thick was then sputtered onto the prestretched PDMS substrate. The relaxation of the prestrain in the PDMS compresses the $\mathrm{Au} / \mathrm{Pd}$ thin film and leads to thin films buckle in a periodic shape with buckling wavelength in the micron range.

Figure 1(b) shows the tilted scanning electron microscope (SEM) image of the periodically buckled $\mathrm{Au} / \mathrm{Pd}$ film, and Fig. 1(c) is an enlarged image of the buckled profile, for $30 \%$ prestrain. The cracks along the prestrain directions in the $\mathrm{Au} / \mathrm{Pd}$ film in Fig. 1(b) are caused by the lateral tensile deformation of PDMS during the relaxation process due to the Poisson effect. Figure 1(d) is a tapping mode atomic force microscope (AFM) image of the buckled thin films. The wavelength $d$ and amplitude $A$ are 1.21 and $0.19 \mu \mathrm{m}$, respectively. These values agree well with the analytical analysis [Eqs. (1) and (2)] if the following material parameters are used, $E_{\mathrm{f}}=80 \mathrm{GPa}, E_{\mathrm{s}}=2 \mathrm{MPa}, h_{\mathrm{f}}=11 \mathrm{~nm}, \nu_{\mathrm{f}}=0.3$, and $\nu_{\mathrm{s}}=0.49$. The buckling profile can be adjusted by applying a mechanical strain $\varepsilon_{\text {applied }}$ and the wavelength and amplitude change accordingly

$$
\begin{aligned}
& d=\frac{2 \pi h_{\mathrm{f}}\left(1+\varepsilon_{\text {applied }}\right)}{\left(1+\varepsilon_{\text {pre }}\right)\left(1+\varepsilon_{\text {applied }}+\zeta\right)^{1 / 3}}\left[\frac{E_{\mathrm{f}}\left(1-\nu_{\mathrm{s}}^{2}\right)}{3 E_{\mathrm{s}}\left(1-\nu_{\mathrm{f}}^{2}\right)}\right]^{1 / 3}, \\
& A=h \frac{\sqrt{\left(\varepsilon_{\text {pre }}-\varepsilon_{\text {applied }}\right) / \varepsilon_{\mathrm{c}}-1}}{\sqrt{\left(1+\varepsilon_{\text {pre }}\right)}\left(1+\varepsilon_{\text {applied }}+\zeta\right)^{1 / 3}},
\end{aligned}
$$

where $\zeta=5\left(\varepsilon_{\text {pre }}-\varepsilon_{\text {applied }}\right)\left(1+\varepsilon_{\text {pre }}\right) / 32$. This provides a means to tune the grating period by simple mechanical stretching or

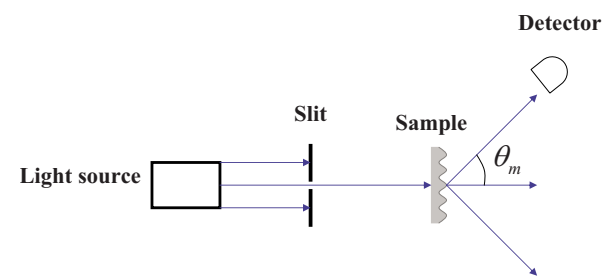

FIG. 2. (Color online) Schematic experimental setup for the stretchable diffraction grating measurement.

releasing thus enabling a spectral shift rather than only altering the intensity of the diffracted light. ${ }^{5}$ The grating period will be predominately determined by the modulus ratio between PDMS and Au/Pd film $\left(E_{\mathrm{f}} / E_{\mathrm{s}}\right)$, the prestrain $\left(\varepsilon_{\text {pre }}\right)$ and applied strain $\left(\varepsilon_{\text {applied }}\right)$ on PDMS, as well as the thickness of the thin $\mathrm{Au} / \mathrm{Pd}$ layer $\left(h_{\mathrm{f}}\right)$. The thickness of PDMS will not have significant effect since the typical thickness ratio of the PDMS and Au/Pd film is on the order of $10^{4}$ and PDMS is transparent to visible light.

The diffraction properties of the tunable gratings have been investigated. The schematic experimental setup is shown in Fig. 2. The light source comes from a xenon arc lamp, with output wavelength ranging from 200 to $2500 \mathrm{~nm}$. A fiber-coupled spectroradiometer was placed at the angle of $\theta_{m}=20^{\circ}$ to detect the transmitted light. Source light passing through a slit was aligned normal to the grating sample. The incident light was therefore diffracted by the grating at a range of transmission angles, according to Eq. (3). Given unchanged angle of incidence $\left(\theta_{\mathrm{i}}=0^{\circ}\right)$ and a fixed angle of diffraction $\left(\theta_{m}=20^{\circ}\right)$, Eq. (3) can be simplified to $m \lambda / d$ $=$ constant. As the period of the grating $d$ is tuned by mechanically stretch or compression [based on Eq. (4)], the wavelength of the first order diffraction light at $\theta_{m}$ changes accordingly.

Measurement of wavelength shift of the transmittance diffraction is performed by fixing the detector and only tuning the applied strain on the grating. At zero applied strain, where diffraction grating has the highest amplitude and shortest period, dispersed color from grating order, $m$, as high as 5 can be clearly observed, with the first order observed with the strongest intensity. With the stretching of the grating, bands from these different orders all move simultaneously. The first order of the diffraction light has significant wavelength shift while stretching the grating from 0 to a maximum of $30 \%$ applied strain, defined by the prestrain of PDMS. As the grating is stretched, inducing an increase in the grating period, the wavelength of the diffraction light entering the detector increases. The peak wavelength of the first order transmittance diffraction light was initially around $418 \mathrm{~nm}$ without any applied strain, and that increases to 503 $\mathrm{nm}$ at the applied strain of $27.5 \%$, as shown in Figs. 3(a) and 3(b). The fine peaks in Fig. 3(a) come primarily from detector noise. It is also noticed that as the applied strain increases up to $30 \%$, at which level the $\mathrm{Au} / \mathrm{Pd}$ film becomes flattened, the \pm 1 st order diffraction coincides with the 0 th order. The wavelength tunability is around $85 \mathrm{~nm}$, which is clearly indicated by the color shifting from violet to green, thus demonstrating as a widely tunable optical grating.

The intensity of the transmittance diffraction light entering the detector at different applied strain levels also varies, due to the change in amplitude of the grating with applied strain. Similar behavior has also been observed in the previ- 


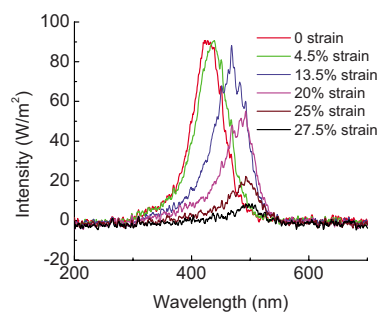

(a)

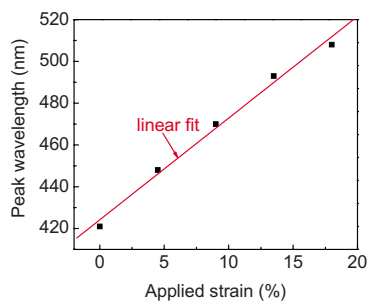

(c)

FIG. 3. (Color online) The diffraction properties of buckled Au/Pd films: (a) the measured intensities of the first order diffraction show the light wavelength shift of $85 \mathrm{~nm}$ assuming the detector is fixed; (b) the peak wavelength of the diffraction light (left axis) increases linearly with the applied strain while the intensity (right axis) decreases while stretching the grating. (c) The peak wavelength of the diffraction light of buckled ITO film increases linearly with the applied strain.

ous report. ${ }^{5}$ As the grating is stretched, the amplitude of the sinusoidal grating also decreases, which contributes to the decrease in the diffraction intensity as plotted in Fig. 3(b). The closer the stretching to the $30 \%$ prestrain level, the faster the amplitude decreases, and it eventually reduces to close zero intensity at $30 \%$ stretching as at this stage grating amplitude becomes zero. To avoid the extreme condition in which the intensity drops to zero, one can operate such a stretchable grating in the low strain region which could provide large grating amplitude, lower stress for sustained use, and yet large enough range for tuning.

The surface plasmonic effect that may contribute to tuning phenomenon was examined. Reemitted photons due to plasmonic effect in the Au film are scattered light in nature with random orientation, therefore it should not demonstrate the diffraction behavior that can be described by standard gratings. Furthermore, tunable gratings have also been fabricated using other materials as buckled stiff thin films. Buckled indium tin oxide (ITO) thin films (20 nm thick) are deposited on a PDMS substrate with $20 \%$ prestrain by following the same procedure described in Fig. 2. The grating measurement is conducted and the similar tuning behavior is shown in Fig. 3(c) in the visible range. Therefore, we conclude that Au surface plasmonic effect does not contribute significantly to the tunable grating while the change of the buckling period is the major reason.

In conclusion, tunable diffractive gratings have been realized based on sinusoidally buckled thin film on transparent and elastomeric PDMS substrates. The tunability is achieved by stretching of the periodic wavy thin film/PDMS system, thereby altering the grating period. The fabricated tunable grating shows $85 \mathrm{~nm}$ wavelength shift of the first order diffraction by stretching the grating up to $30 \%$ of its original length. Gratings with different tunability could be easily designed and fabricated for promising applications such as optical switches, tunable light scanners, or strain sensors.

H.J. acknowledges the support from NSF under Grant No. CMMI-0700440, and H.Y. acknowledges the support from NSF under Grant No. ECCS-0926017.

${ }^{1}$ P. Äyräs, J. T. Rantala, S. Honkanen, S. B. Mendes, and N. Peyghambarian, Opt. Commun. 162, 215 (1999).

${ }^{2}$ C. W. Wong, Y. Jeon, G. Barbastathis, and S. Kim, Appl. Opt. 42, 621 (2003).

${ }^{3}$ C. S. Gudeman, C. B. Carlisle, J. Hunter, R. W. Corrigan, R. J. Monteverde, and J. I. Trisnadi, Proc. SPIE 4653, 56 (2002).

${ }^{4}$ A. A. Yasseen, S. W. Smith, F. L. Merat, and M. Mehregany, IEEE J. Sel. Top. Quantum Electron. 75, 5 (1999).

${ }^{5}$ C. Harrison, C. M. Stafford, W. Zhang, and A. Karim, Appl. Phys. Lett. 85, 4016 (2004).

${ }^{6}$ N. Bowden, S. Brittain, A. G. Evans, J. W. Hutchinson, and G. M. Whitesides, Nature (London) 393, 146 (1998).

${ }^{7}$ S. P. Lacour, J. Jones, S. Wagner, T. Li, and Z. G. Suo, Proc. IEEE 93, 1459 (2005).

${ }^{8}$ D. Y. Khang, H. Q. Jiang, Y. Huang, and J. A. Rogers, Science 311, 208 (2006)

${ }^{9}$ C. Yu, C. Masarapu, J. P. Rong, B. Q. Wei, and H. Jiang, Adv. Mater. 21, 4793 (2009).

${ }^{10}$ C. Yu, Z. Wang, H. Yu, and H. Jiang, Appl. Phys. Lett. 95, 141912 (2009).

${ }^{11}$ C. M. Stafford, C. Harrison, K. L. Beers, A. Karim, E. J. Amis, M. R. Vanlandingham, H. C. Kim, W. Volksen, R. D. Miller, and E. E. Simonyi, Nature Mater. 3, 545 (2004).

${ }^{12}$ W. T. S. Huck, N. Bowden, P. Onck, T. Pardoen, J. W. Hutchinson, and G. M. Whitesides, Langmuir 16, 3497 (2000).

${ }^{13}$ R. Huang, J. Mech. Phys. Solids 53, 63 (2005).

${ }^{14}$ H. Q. Jiang, D. Y. Khang, H. Y. Fei, H. Kim, Y. G. Huang, J. L. Xiao, and J. A. Rogers, J. Mech. Phys. Solids 56, 2585 (2008).

${ }^{15}$ H. Jiang, D.-Y. Khang, J. Song, Y. Sun, Y. Huang, and J. A. Rogesr, Proc. Natl. Acad. Sci. U.S.A. 104, 15607 (2007).

${ }^{16}$ D. H. Raguin and G. M. Morris, Appl. Opt. 32, 1154 (1993). 